\title{
Pemetrexed pharmacokinetics and pharmacodynamics in a phase I/II study of doublet chemotherapy with vinorelbine: implications for further optimisation of pemetrexed schedules
}

\author{
KM Li', LP Rivory ${ }^{2}$ and SJ Clarke*,3 \\ 'Discipline of Pharmacology, Faculty of Medicine, Bosch Institute, School of Medical Sciences, University of Sydney, Sydney, New South Wales 2006, \\ Australia; ${ }^{2}$ Johnson and Johnson Research Pty. Ltd, Strawberry Hills, New South Wales 2012, Australia; ${ }^{3}$ Department of Medicine, Concord Hospital, \\ Concord, New South Wales 2137 , Australia
}

\begin{abstract}
The purpose of this study was to investigate the utility of plasma pharmacokinetic and pharmacodynamic measures including plasma deoxynucleosides, homocysteine and methylmalonic acid concentrations in understanding the time course and extent of the inhibition of thymidylate synthase (TS) by pemetrexed in the context of a phase I/II combination study with vinorelbine. Eighteen patients received supplementation with folic acid and Vitamin $B_{12} \mid$ week before beginning treatment with pemetrexed and vinorelbine administered in a dose-escalating manner on a 21 -day cycle. Heparinised blood samples were collected from consenting patients in the first cycle for pharmacokinetic analyses and in the first two cycles for determination of plasma thymidine, deoxyuridine, homocysteine and methylmalonic acid concentrations. These values were correlated with response and toxicity. Plasma deoxyuridine was used as a measure of TS inhibition, and concentrations of deoxyuridine were significantly elevated relative to baseline on days I $(P<0.0 \mathrm{I}), 2(P<0.00 \mathrm{I})$ and $3(P<0.05)$ after treatment at all pemetrexed dose levels $\left(400-700 \mathrm{mg} \mathrm{m}^{-2}\right)$. The magnitude of deoxyuridine elevation correlated with pemetrexed area under the plasma concentration-time curve $(A \cup C)\left(r^{2}=0.23, P<0.05\right)$. However, deoxyuridine concentrations returned to baseline between 8 and 15 days after treatment with pemetrexed, suggesting that inhibition of TS was not durable. Pemetrexed AUC correlated with the percentage decline (relative to baseline) in both platelets $\left(r^{2}=0.58, P<0.00 I\right)$ and leucocytes $\left(r^{2}=0.26, P<0.05\right)$ at day 8 . Baseline homocysteine was also significantly correlated with these measures of haematological toxicity $\left(r^{2}=0.37, P<0.01\right.$ and $r^{2}=0.39, P<0.01$, respectively). In addition, there was a significant reduction of plasma homocysteine on days $8(P<0.005)$ and $15(P<0.05)$ in cycle I compared to baseline values. The results suggest that the TS inhibitory effects of pemetrexed are short-lived and make the case for a more frequent schedule of administration such as every 2 weeks. The lack of protracted TS inhibition may be due to concomitant vitamin administration, and this may be the mechanism by which vitamins prevent life-threatening toxicity from pemetrexed. Baseline homocysteine concentration remains a predictive marker for haematological toxicity even following folate supplementation.
\end{abstract}

British Journal of Cancer (2007) 97, I07I-1076. doi: I0.1038/sj.bjc.6603995 www.bjcancer.com

Published online 2 October 2007

(c) 2007 Cancer Research UK

Keywords: thymidylate synthase inhibition; pemetrexed; anti-folates; pharmacodynamic markers; plasma deoxynucleosides; homocysteine

Pemetrexed, a multitargeted anti-folate, has shown activity in various tumours, especially mesothelioma and non-small-cell lung cancer (NSCLC) for which it is routinely used (Adjei, 2003). Pemetrexed inhibits at least three of the enzymes involved in folate metabolism, and pyrimidine and purine biosynthesis, as shown in Figure 1. These include thymidylate synthase (TS), dihydrofolate reductase (DHFR) and glycinamide ribonucleotide formyltransferase. However, inhibition of TS has been shown to be the primary mechanism of action of pemetrexed (Grindey et al, 1992). TS, an $N^{5}, N^{10}$-methylene tetrahydrofolate (methylene-THF)-dependent enzyme, catalyses the reductive methylation of deoxyuridylate

*Correspondence: Professor SJ Clarke; E-mail: sclarke@med.usyd.edu.au Received 5 June 2007; revised 16 August 2007; accepted 29 August 2007; published online 2 October 2007
(dUMP) to deoxythymidylate, the rate-limiting step in the de novo synthesis of deoxythymidine triphosphate. Inhibition of DHFR by pemetrexed reduces the regeneration of the essential coenzyme methylene-THF thereby further enhancing the TS inhibitory effects.

Inhibition of TS in tissues leads to intracellular accumulation of dUMP and subsequent efflux of deoxyuridine (dUrd) into the circulation. Accumulation of dUMP occurs as a direct consequence of TS inhibition and also as a result of increased activity of other enzymes, including ribonucleotide reductase (RR) and deoxycytidylate (dCMP) deaminase (Maybaum et al, 1981). A number of studies have indicated that plasma dUrd is an important pharmacodynamic (PD) marker of TS inhibition (Mitchell et al, 1997; de Jonge et al, 2002; Ford et al, 2002; Rees et al, 2003) especially with the use of the TS inhibitors AG337 (Nolatrexed) 
(Jodrell et al, 1999; Estlin et al, 2001), ZD9331 (de Jonge et al, 2002; Ford et al, 2002; Rees et al, 2003) and ZD1964 (raltitrexed) (Ford et al, 2002; Horton et al, 2005); however, similar studies have not been performed with pemetrexed.

Inhibition of TS may be offset by thymidine (TdR) salvage pathways, and administration of exogenous TdR has been shown to rescue tumour cell lines from pemetrexed-induced cytotoxicity (Smith et al, 1999). Therefore, basal plasma TdR concentrations might be predictive for response and toxicity with TS inhibitors. However, correlative assessments of $\mathrm{TdR}$ and toxicity have not previously been possible because of the lack of an appropriately sensitive and specific assay. We have recently developed and validated a liquid chromatography mass spectrometry (LC-MS) assay that has demonstrated that human plasma TdR concentrations are much lower than previously reported (Li et al, 2003).

Myelosuppression is the principal toxicity following treatment with pemetrexed with $50 \%$ of patients experiencing grade $3 / 4$ toxicity (Hanauske et al, 2001). Early in the preclinical evaluation of pemetrexed, it became apparent that functional folate status was an important factor in the activity and toxicity of this compound (Worzalla et al, 1998). Methionine synthase, the enzyme which catalyses the conversion of homocysteine (Hcy) to methionine (Met), requires $N^{5}$-methyltetrafolate (methyl-THF) as a methyl group donor and vitamin $\mathrm{B}_{12}$ for this reaction to proceed. When methyl-THF and vitamin $B_{12}$ levels are low, the conversion of Hcy to Met decreases, resulting in increased concentrations of Hcy (Figure 1). Thus, Hcy can be used as a marker for overall folate status in the body. Likewise, vitamin $B_{12}$ is a vital cofactor for methylmalonyl coenzyme A mutase, and the level of methylmalonyl acid (MMA) increases as a result of vitamin $B_{12}$ deficiency (Savage et al, 1994; Lucock et al, 1999). It has been shown that plasma Hcy and MMA levels are important markers in predicting severe toxicity with pemetrexed (Niyikiza et al, 2002).

Administration of folic acid ( $0.5 \mathrm{mg}$ per mouth each day) and vitamin $B_{12}$ ( $1 \mathrm{mg}$ by intramuscular injection every 9 weeks) commencing 1 week prior to treatment with pemetrexed has been shown to reduce the incidence of life-threatening toxicity, especially myelosuppression (Bunn et al, 2001). However, the use of vitamin prophylaxis permits higher doses of pemetrexed to be safely used, creating some uncertainty about the optimal schedule and dose of this agent in the post-vitamin era. Consequently, a comparison of the clinical pharmacokinetics (PK) of pemetrexed with markers of its PD effects could clarify these issues of optimal dose and schedule.

Previously, we have reported that the combination of pemetrexed and vinorelbine is well tolerated and shows activity as firstline treatment in advanced NSCLC patients (Clarke et al, 2005). The objective of the current study was to further investigate the PD of TS inhibitory effects by analysing plasma dUrd and TdR concentrations and correlating them with toxicity in these patients. By including a PK analysis of pemetrexed, another objective was to assess possible PK-PD correlations during the first course of treatment. Furthermore, we wished to correlate drug-induced toxicities with functional folate status in vitamin-supplemented patients.

\section{MATERIALS AND METHODS}

Eighteen patients from the phase I/II study of pemetrexed and vinorelbine at the Sydney Cancer Centre at Royal Prince Alfred

Table I Patient demographics and clinical characteristics $(n=18)$

\begin{tabular}{lc}
\hline Parameter & No. of patients (\%) \\
\hline Male & $13(72 \%)$ \\
Median age, years (range) & $58(40-72)$ \\
Performance status & \\
0 & $5(28 \%)$ \\
I & $13(72 \%)$ \\
Disease status & \\
Metastatic & $17(94 \%)$ \\
Locally advanced & $1(6 \%)$ \\
Prior adjuvant chemotherapy & 0 \\
Prior chemotherapy for metastatic disease & $12(67 \%)$ \\
Prior chemotherapy for locally advanced disease & $1(6 \%)$ \\
Prior radiotherapy & $5(27 \%)$ \\
\hline
\end{tabular}

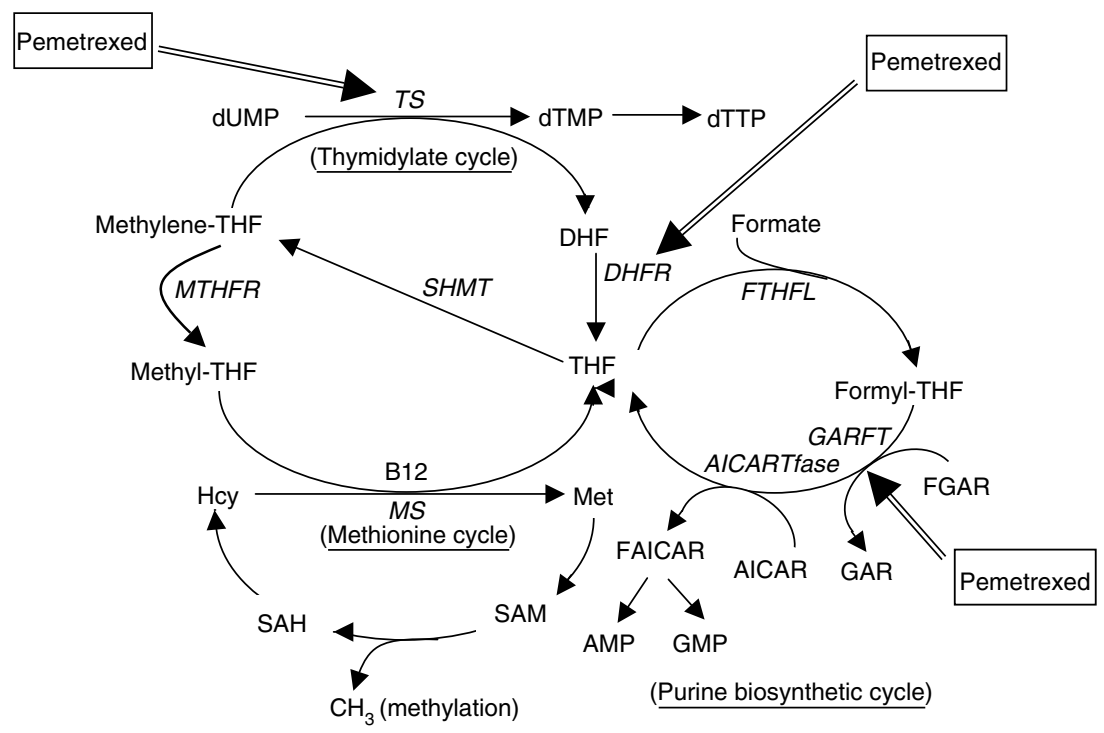

Figure I Folate involved in biosynthesis of pyrimidine, purine and methionine. AICARTfase, aminoimidazole carboxamide ribonucleotide transformylase; AMP, adenosine monophosphate; GMP, guanosine monophosphate; FAICAR, formylaminoimidazole ribonucleotide; FGAR, formylglycinamide ribonucleotide; FTHFL, formate-tetrahydrofolate ligase; MTHFR, methyltetrahydrofolate reductase; SAM, S-adenosylmethionine; SAH, S-adenosylhomocysteine; SHMT, serine hydroxymethyltransferase. 
Hospital agreed to participate in this substudy. The demographics and clinical characteristics of the patients are summarised in Table 1. Eligibility criteria for patient recruitment were NSCLC with at least aged 18 years with minimum estimated 12 weeks life expectancy and performance status 0-2; good bone marrow reserve (platelets $>100 \times 10^{9} \mathrm{l}^{-1}$, haemoglobin $>90 \mathrm{gl}^{-1}$, absolute granulocyte count $>2.0 \times 10^{9} 1^{-1}$ ); adequate hepatic function (bilirubin $<1.5 \times$ upper limit of normal (ULN), transaminase $<3.0 \times \mathrm{ULN}$ (unless liver metastases present)); and adequate renal function (creatinine clearance $>0.75 \mathrm{ml} \mathrm{s}^{-1}$, albumin $>25 \mathrm{gl}^{-1}$ ). Exclusion criteria were symptomatic brain metastases, uncontrolled pleural or peritoneal effusions, and inability to interrupt non-steroidal anti-inflammatory drugs. The study was approved by the Human Ethics Research Committees of the Central Sydney Area Health Service and the University of Sydney.

\section{Treatment}

The patients received $400-700 \mathrm{mg} \mathrm{m}^{-2}$ pemetrexed (Alimta ${ }^{\odot}$, Eli Lilly and Company, Indianapolis, IN, USA) as a 10-min infusion on day 1 of a 21-day cycle. Vinorelbine was infused at 15$30 \mathrm{mg} \mathrm{m}^{-2}$ (approximately 6-10 min infusion) on days 1 and 8 of a 21-day cycle. On day 1, the vinorelbine infusion began $30 \mathrm{~min}$ after the end of the pemetrexed infusion. This 21-day period defined one cycle of therapy. All patients received supplementation with folic acid (500 $\mu \mathrm{g}$ p.o., daily) and vitamin $\mathrm{B}_{12}(1000 \mu \mathrm{g}$ i.m., every 9 weeks) commencing 1 week before the first cycle and continuing until the discontinuation of chemotherapy.

\section{Blood samples collection procedure}

For the PK study, heparinised blood samples were collected at time 0 (baseline), $9.5 \mathrm{~min}$ (end of pemetrexed infusion), 15, 30, $50 \mathrm{~min}$ (end of vinorelbine infusion), $1 \mathrm{~h}, 1 \mathrm{~h} 20 \mathrm{~min}, 2,4,8,24,48$ and $72 \mathrm{~h}$, for measurement of plasma pemetrexed and vinorelbine concentrations over a 72-h period following the start of pemetrexed administration in first cycle. In addition, blood samples were also collected from patients on days $0,1,2,3,4,8$ and 15 of the first cycle and days 0,8 and 15 of the second cycle for measurement of TdR, dUrd, Hcy and MMA concentrations. Whole blood was collected into prechilled heparinised blood tubes and placed on ice immediately. Plasma was rapidly separated from cellular components by centrifuging at $3300 \mathrm{~g}$ at $4^{\circ} \mathrm{C}$ for $15 \mathrm{~min}$. Plasma was then stored at $-80^{\circ} \mathrm{C}$ and thawed just prior to analysis.

\section{Pharmacokinetic analysis}

Plasma pemetrexed concentrations were analysed at Taylor Technology Inc. (Princeton, NJ, USA) using a validated liquid chromatography electrospray ionisation with tandem mass spectrometric detection method over the concentration ranges of 10-2000 and 1000-200000 $\mathrm{ng} \mathrm{m}^{-1}$ (Latz et al, 2006). Samples were analysed for vinorelbine using a validated HPLC-fluorescence detection method over the concentration range of $2-500 \mathrm{ng} \mathrm{ml}^{-1}$ by Bioanalytical Laboratory Service (PPD Development, Richmond, VA, USA).

\section{Measurement of plasma TdR, dUrd, Hcy and MMA levels}

Plasma TdR and dUrd levels were analysed using a validated LC-MS method, which we previously published (Li et al, 2003, 2005). Briefly, plasma samples were extracted with strong anionexchange solid-phase extraction columns followed by HPLC separation and atmospheric pressure chemical ionisation mass spectrometry detection in a selected-ion monitoring mode. Plasma Hcy and MMA were analysed by Douglas Hanley Moir Laboratory (Sonic Clinical Trial Centre, North Ryde, NSW, Australia).

\section{Statistical analysis}

PK parameters were evaluated by compartmental and multiple compartmental methods for pemetrexed and vinorelbine, respectively (Lee and Amidon, 1996). The area under the plasma concentration-time curve (AUC) was determined using the linear trapezoidal method with extrapolation to infinity. Total plasma clearance values of pemetrexed and vinorelbine for each patient were calculated by dividing the dose administered by the AUC. The values for plasma nucleosides, Hcy and methylmalonic acid at each time point are expressed as mean \pm s.d. Statistical analysis of data was achieved by Student's $t$-tests (paired) and regression correlation (GraphPad Prism). $P<0.05$ was considered to be statistically significant.

\section{RESULTS}

\section{Pharmacokinetic analysis}

The full PK for the phase I/II trial have been published previously (Clarke et al, 2005). The PK parameters for pemetrexed and vinorelbine for the subgroup of patients included in this study are summarised in Tables 2 and 3, respectively.

\section{Plasma TdR, dUrd, Hcy and MMA analysis}

Plasma dUrd and TdR concentrations during the first and second cycles of treatment are shown in Figure 2. Plasma dUrd concentrations were significantly elevated on days 1,2 and 3 after treatment at all pemetrexed dose levels. However, there was no significant change of plasma TdR following pemetrexed treatment. Mean pretreatment plasma dUrd and TdR concentrations of all 18 patients were $66.9 \pm 30.8$ and $11.7 \pm 6.36 \mathrm{nmoll}^{-1}$, respectively. Plasma dUrd in treated patients was significantly increased compared to baseline on days $1\left(172 \mathrm{nmoll}^{-1}, 255 \%, P<0.01\right)$, $2\left(210 \mathrm{nmoll}^{-1}, 315 \%, P<0.001\right)$ and $3\left(170 \mathrm{nmoll}^{-1}, 252 \%\right.$,

Table 2 Summary of PK parameters for pemetrexed

\begin{tabular}{lcccc}
\hline & \multicolumn{4}{c}{ Pemetrexed expressed as mean \pm s.d. } \\
\hline Dose $\left(\mathrm{mg} \mathrm{m}^{-2}\right)$ & 400 & 500 & 600 & 700 \\
No. of patients & 6 & 5 & 3 & 4 \\
$A \cup C_{0-\infty}\left(\mathrm{h} \mu \mathrm{g} \mathrm{ml}^{-1}\right)$ & $130 \pm 36.4$ & $194 \pm 75.8$ & $187 \pm 30.5$ & $216 \pm 29.3$ \\
$C_{\max }\left(\mu g \mathrm{ml}^{-1}\right)$ & $89.1 \pm 25.2$ & $134 \pm 29.4$ & $105 \pm 10.5$ & $131 \pm 18.4$ \\
$\mathrm{CL}_{p}\left(1 \mathrm{~h}^{-1} \mathrm{~m}^{-2}\right)$ & $3.29 \pm 0.96$ & $2.59 \pm 0.94$ & $3.28 \pm 0.58$ & $3.32 \pm 0.48$ \\
$t_{(1 / 2)}(\mathrm{h})$ & $2.57 \pm 1.13$ & $2.58 \pm 1.47$ & $1.91 \pm 0.49$ & $3.00 \pm 0.85$ \\
$V_{\mathrm{ss}}\left(1 \mathrm{~m}^{-2}\right)$ & $10.3 \pm 3.71$ & $7.19 \pm 1.68$ & $8.07 \pm 1.41$ & $10.9 \pm 2.87$ \\
\hline
\end{tabular}

$\mathrm{AUC}=$ area under the concentration-time curve; $\mathrm{CL}_{\mathrm{p}}=$ plasma clearance; $C_{\max }=$ maximum plasma concentration; $\mathrm{PK}=$ pharmacokinetic; s.d. = standard deviation; $t_{(1 / 2)}=$ terminal elimination half-life; $V_{\mathrm{ss}}=$ steady-state volume distribution.

Table 3 Summary of PK parameters for vinorelbine

\begin{tabular}{lccc}
\hline & \multicolumn{3}{c}{ Vinorelbine expressed as mean \pm s.d. } \\
\hline Dose $\left(\mathrm{mg} \mathrm{m}^{-2}\right)$ & 15 & 23 & 30 \\
No. of patients & 7 & 3 & 8 \\
$A \cup C_{0-\infty}\left(\mathrm{h} \mu \mathrm{g} \mathrm{m}^{-1}\right)$ & $0.88 \pm 0.60$ & $1.78 \pm 0.38$ & $2.04 \pm 1.03$ \\
$C_{\max }\left(\mu \mathrm{g} \mathrm{m}^{-1}\right)$ & $1.48 \pm 1.13$ & $2.91 \pm 0.14$ & $2.98 \pm 1.83$ \\
$\mathrm{CL}_{\mathrm{p}}\left(\mathrm{Ih}^{-1} \mathrm{~m}^{-2}\right)$ & $27.4 \pm 22.9$ & $12.9 \pm 0.62$ & $18.7 \pm 9.73$ \\
$t_{(\mathrm{I} / 2)}(\mathrm{h})$ & $48.3 \pm 15.2$ & $36.5 \pm 8.21$ & $42.0 \pm 11.6$ \\
$V_{\mathrm{ss}}\left(\mathrm{Im}^{-2}\right)$ & $1067 \pm 543$ & $167 \mathrm{I} \pm 373$ & $1649 \pm 1240$ \\
\hline
\end{tabular}

$A \cup C=$ area under the concentration-time curve; $C_{p}=$ plasma clearance; $C_{\max }=$ maximum plasma concentration; $\mathrm{PK}=$ pharmacokinetic; s.d. = standard deviation; $t_{(1 / 2)}=$ terminal elimination half-life; $V_{s s}=$ steady-state volume distribution. 


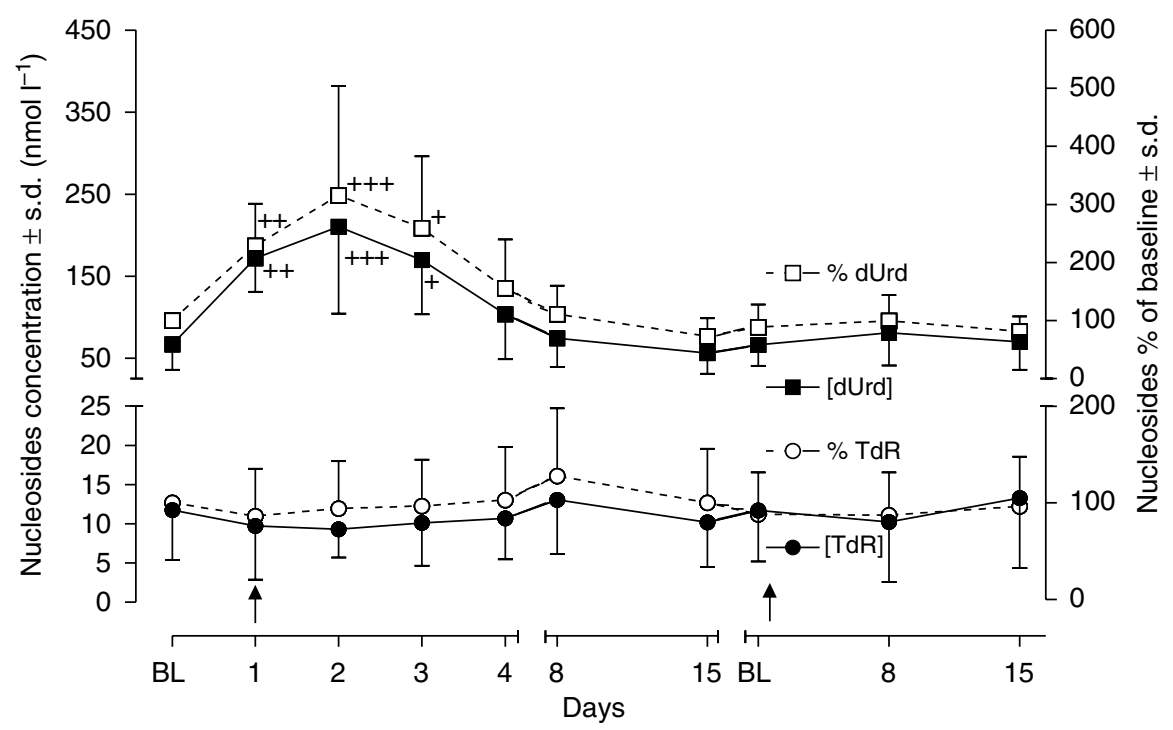

Figure 2 Plasma nucleoside concentrations and \% of baseline changes following pemetrexed-vinorelbine treatment. Data are means \pm s.d. ${ }^{+} P<0.05$, $+P<0.01$ and $+++P<0.001$ when compared with corresponding baseline values.

A

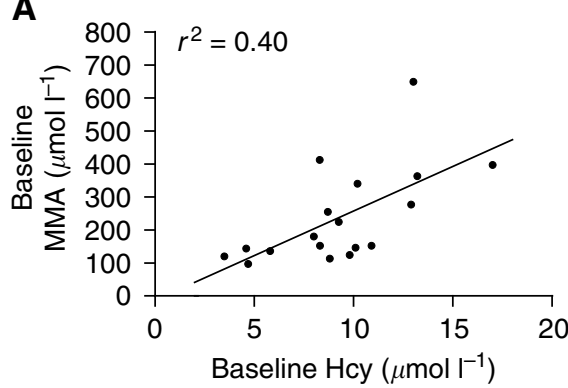

B

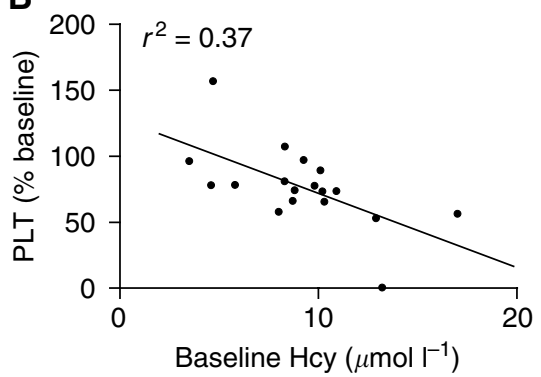

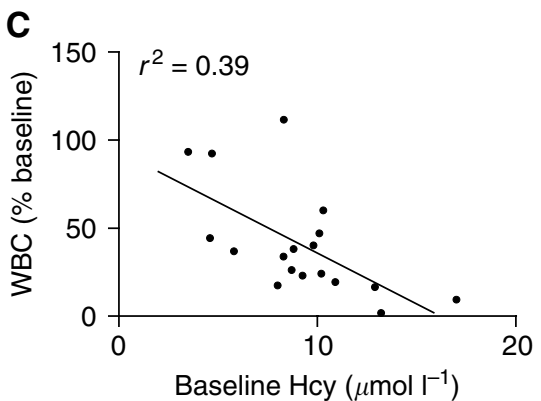

Figure 3 Correlation between baseline Hcy and $(\mathbf{A})$ baseline MMA $\left(r^{2}=0.40, P<0.01\right),(\mathbf{B}) \mathrm{PLT}\left(r^{2}=0.37, P<0.0 \mathrm{I}\right)$ and $(\mathbf{C}) \mathrm{WBC}\left(r^{2}=0.39, P<0.0 \mathrm{I}\right)$ on day 8 of treatment cycle I.

$P<0.05)$ when averaged for all doses. By day 4 , the plasma dUrd levels remained elevated relative to baseline, although this did not reach statistical significance $\left(103 \mathrm{nmoll}^{-1}, 154 \%\right.$, NS). Deoxyuridine concentrations returned to baseline by day 8 . In contrast, mean plasma TdR concentrations were 86,94 and $96 \%$ of baseline on days 1, 2 and 3, and this was not significantly different to baseline values.

The basal Hcy and MMA concentrations were $9.3 \pm 2.95 \mu \mathrm{moll}^{-1}$ (range: $4.72-17.2$ ) and $224 \pm 106 \mathrm{nmoll}^{-1}$ (range: 97-412), respectively for the 18 patients. Most of the patients (17 out of 18) had Hcy values within the normal range $\left(5-13.9 \mu \mathrm{moll}^{-1}\right)$, and only one patient had elevated Hcy $\left(17.2 \mu \mathrm{moll}^{-1}\right)$. Five patients $(5$ out of 18) had abnormally high MMA levels and these ranged from 340 to $412 \mathrm{nmoll}^{-1}$ (normal range: $73-271 \mathrm{nmoll}^{-1}$ ). Baseline Hcy was significantly correlated with baseline MMA $\left(r^{2}=0.40\right.$, $P<0.01)$, but only baseline Hcy correlated with the reduction (\% baseline) of both platelets $\left(r^{2}=0.37, P<0.01\right)$ and leucocytes $\left(r^{2}=0.39, P<0.01\right)$ on day 8 of treatment cycle 1 (Figure 3$)$. There was a significant reduction in plasma Hcy on days $8(P<0.005)$ and $15(P<0.05)$ compared to baseline values as shown in Figure 4. There was also a trend towards reduction in MMA levels after treatment; however, this did not achieve statistical significance. There was no correlation between either baseline Hcy or MMA concentrations with the maximum elevation of plasma dUrd in treatment cycle 1 .

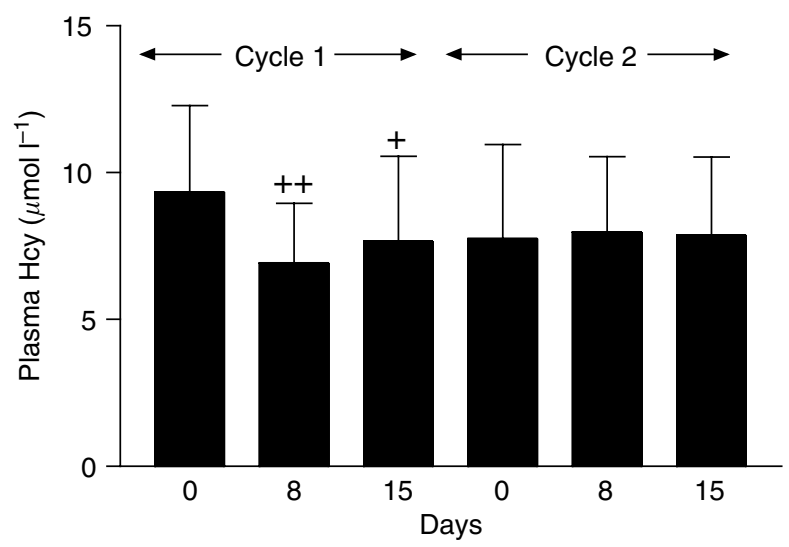

Figure 4 Plasma Hcy concentrations in treatment cycles I and 2. Data are means \pm s.d. ${ }^{+} P<0.05$ and ${ }^{+}+P<0.005$ when compared with baseline concentrations.

The total body exposure of pemetrexed (AUC) was significantly correlated with reduction ( $\%$ baseline) of platelets $\left(r^{2}=0.58\right.$, $P<0.001)$ and leucocytes $\left(r^{2}=0.26, P<0.05\right)$ on day 8 and maximum increase $\left(\%\right.$ baseline) of $\mathrm{dUrd}\left(r^{2}=0.23, P<0.05\right)$ on 

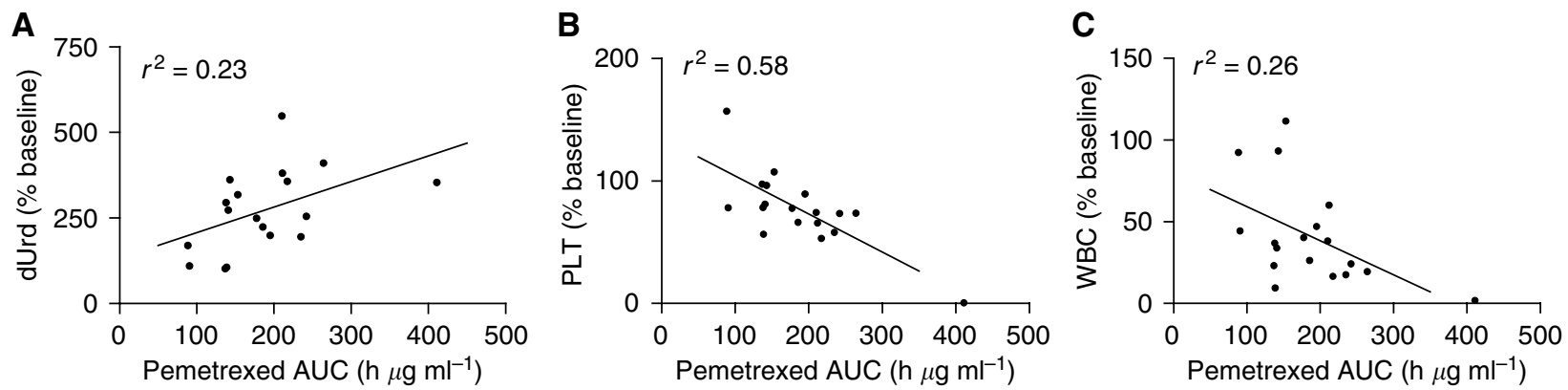

Figure 5 Correlation between total body exposure of pemetrexed (AUC) and $(\mathbf{A}) d U r d$ on day $2\left(r^{2}=0.23, P<0.05\right),(\mathbf{B}) P L T\left(r^{2}=0.58, P<0.00 I\right)$ and $(\mathbf{C})$ WBC $\left(r^{2}=0.26, P<0.05\right)$ on day 8 of treatment cycle I.

day 2 as shown in Figure 5. However, there was no significant correlation between the change in plasma dUrd and platelet and leucocyte counts on either day 8 or 15.

\section{DISCUSSION}

The objective of this study was to investigate PK-PD aspects of pemetrexed in the setting of a phase I/II combination study. The PK of pemetrexed and vinorelbine were consistent with data obtained with single-agent chemotherapy (Marquet et al, 1992; Leveque and Jehl, 1996; Sabot et al, 1998). There was no evidence for PK interaction between the two drugs. In the current study, measurement of plasma dUrd concentration showed that pemetrexed administered at doses of $400-700 \mathrm{mg} \mathrm{m}^{-2}$ produced consistent elevations in plasma dUrd, suggesting that plasma exposure to pemetrexed was sufficient to induce TS inhibition in patients. Indeed, the current results have demonstrated a clear PK - PD relationship for TS inhibition in these patients (Figure 5). On days 1-3, plasma dUrd levels were significantly higher than baseline, and this may suggest rapid TS inhibition after dosing. The maximum increase of dUrd level was observed on day 2 following which plasma dUrd gradually returned to baseline by day 8, suggesting that TS inhibition was short-lived with this schedule of pemetrexed. This pattern is comparable to previous studies with the classical anti-folate raltitrexed (RTX), which showed dUrd elevations on days $1-3$ and which returned back to baseline within 2 weeks of treatment (Ford et al, 2002; Horton et al, 2005).

Intracellularly, pemetrexed is polyglutamated by a reaction catalysed by folylpolyglutamate synthase. Polyglutamated pemetrexed is approximately 60 -fold more potent in its inhibition of TS than the parent monoglutamate compound (Shih et al, 1998) and is retained intracellularly long term. Therefore, the highly inhibitory polyglutamated forms would be expected to generate long-lived TS inhibition. However, similar to the TS-selective inhibitor RTX, TS inhibition by pemetrexed, as indicated by elevations in plasma dUrd concentrations, was relatively transient. There are a number of possible explanations for this. Firstly, plasma dUrd concentrations may not be sensitive indicators of TS inhibition. However, in a previous study of capecitabine we saw protracted elevation of plasma dUrd concentrations that lasted for the entire course of treatment ( $\mathrm{Li}$ et al, 2007). An alternative explanation is that inhibition of TS by folate-based agents is not durable, in spite of intracellular polyglutamation, and can be competed out by naturally occurring folates once plasma drug levels are reduced. This competition would be enhanced by exogenous administration of folic acid, which could explain why vitamin-supplemented patients do not experience protracted, potentially lethal toxicity.

Regardless of the explanation, these data suggest that other more frequent administration schedules of pemetrexed, such as bi-weekly regimens, should be explored to optimise its antiproliferative effects. Although cell death produced by pemetrexed can be prevented in culture through the addition of exogenous $\mathrm{TdR}$, the plasma concentration of TdR observed in patients is likely to be too low to play a significant role in the modulation of pemetrexed activity through salvage pathways. Therefore, human plasma TdR level does not appear to be a useful PD phenotypic marker for TS inhibition.

Because anti-folates can interfere with the normal metabolic pathway of folates in the body, it might be reasonable to speculate that treatment with pemetrexed would alter folate metabolism and that this would be reflected by an elevation of plasma Hcy concentration. Surprisingly, the current study has shown that plasma Hcy was not elevated but instead decreased in the first 2 weeks following pemetrexed therapy (Figure 4). Indeed, it has been previously reported that plasma Hcy concentrations are not affected by pemetrexed (Niyikiza et al, 2002). However, patients did not receive folate supplementation in that particular study. Therefore, it is likely that the kinetics of reduction of plasma Hcy observed in current study are the direct result of expansion of intracellular folate pools by folate $/ \mathrm{B}_{12}$ supplementation. This is consistent with previous studies that have demonstrated that folate supplementation, with or without additional $B_{12}$ and $B_{6}$, can significantly lower plasma Hcy (Naurath et al, 1995; Refsum et al, 1996; Bronstrup et al, 1998; Homocysteine Lowering Trialists' Collaboration, 1998; Bunn et al, 2001; Billion et al, 2002).

In the present study, a statistically significant relationship between baseline Hcy and MMA was established in patients. This correlation is consistent with similar observations in healthy volunteers as well as a number of patient subset studies, although MMA is usually indicative of cobalamin status whereas Hcy is the preferred marker for folate deficiency (Valik et al, 2004). Indeed, only baseline Hcy was statistically associated with increased risk of neutropenia and thrombocytopenia on day 8 of treatment cycle 1 (Figure 3). This supports the notion that plasma Hcy is a more specific marker for overall functional folate status and indicates that baseline Hcy might be useful in predicting toxicity during pemetrexed therapy or be used as support for increased supplementation (either duration or dose) in individuals in whom Hcy remains elevated after initial supplementation. In addition, it also supports the current use of up-front supplementation with folic acid and $B_{12}$ in patients prior to treatment with pemetrexed.

In summary, the results of this study demonstrate PD changes consistent with TS inhibition and a close PK-PD relationship following pemetrexed-vinorelbine treatment. Baseline Hcy appears to be a better predictive marker than MMA for haematological toxicity. The current results also support the use of vitamin supplementation to ensure normal vitamin $B_{12}$ and folate status to control haematologic toxicity secondary to pemetrexed administration. 


\section{REFERENCES}

Adjei AA (2003) Pemetrexed (Alimta): a novel multitargeted antifolate agent. Expert Rev Anticancer Ther 3: 145-155

Billion S, Tribout B, Cadet E, Queinnec C, Rochette J, Wheatley P, Bataille P (2002) Hyperhomocysteinaemia, folate and vitamin $B_{12}$ in unsupplemented haemodialysis patients: effect of oral therapy with folic acid and vitamin $\mathrm{B}_{12}$. Nephrol Dial Transplant 17: 455-461

Bronstrup A, Hages M, Prinz-Langenohl R, Pietrzik K (1998) Effects of folic acid and combinations of folic acid and vitamin B-12 on plasma homocysteine concentrations in healthy, young women. Am J Clin Nutr 68: $1104-1110$

Bunn P, Paoletti P, Niyikiza C, Rusthoven J, Nelson K, Hanauske AR, Stabler S, Calvert AH, Allen R (2001) Vitamin $B_{12}$ and folate reduce toxicity of Alimta ${ }^{\mathrm{TM}}$ (Pemetrexed Disodium, LY231514, MTA), a novel antifolate/antimetabolite. Proc Am Soc Clin Oncol 20: 300

Clarke SJ, Boyer MJ, Millward M, Underhill C, Moylan E, Yip D, White S, Childs A, Beale P, Latz J (2005) A phase I/II study of pemetrexed and vinorelbine in patients with non-small cell lung cancer. Lung Cancer 49: $401-412$

de Jonge MJ, Punt CJ, Sparreboom A, Planting AS, Peters ME, van De Schraaf J, Jackman A, Smith R, de Mulder PH, Verweij J (2002) Phase I and pharmacologic study of oral ZD9331, a novel nonpolyglutamated thymidylate synthase inhibitor, in adult patients with solid tumors. J Clin Oncol 20: $1923-1931$

Estlin EJ, Pinkerton CR, Lewis IJ, Lashford L, McDowell H, Morland B, Kohler J, Newell DR, Boddy AV, Taylor GA, Price L, Ablett S, Hobson R, Pitsiladis M, Brampton M, Clendeninn N, Johnston A, Pearson AD (2001) A phase I study of nolatrexed dihydrochloride in children with advanced cancer. A United Kingdom children's cancer study group investigation. Br J Cancer 84: 11-18

Ford HER, Mitchell F, Cunningham D, Farrugia DC, Hill ME, Rees C, Calvert AH, Judson IR, Jackman AL (2002) Patterns of elevation of plasma $2^{\prime}$-deoxyuridine, a surrogate marker of thymidylate synthase (TS) inhibition, after administration of two different schedules of 5-fluorouracil and the specific TS inhibitors raltitrexed (Tomudex) and ZD9331. Clin Cancer Res 8: $103-109$

Grindey GB, Shih C, Barnett CJ (1992) LY231514, a novel pyrrolopyrimidine antifolate that inhibits thymidylate synthase (TS). Proc Am Assoc Cancer Res Annu Meet 33: 411

Hanauske AR, Chen V, Paoletti P, Niyikiza C (2001) Pemetrexed disodium: a novel antifolate clinically active against multiple solid tumors. Oncologist 6: $363-373$

Homocysteine Lowering Trialists' Collaboration (1998) Lowering blood homocysteine with folic acid based supplements: meta-analysis of randomised trials. BMJ 316: $894-898$

Horton TM, Blaney SM, Langevin A-M, Kuhn J, Kamen B, Berg SL, Bernstein M, Weitman S (2005) Phase I trial and pharmacokinetic study of raltitrexed in children with recurrent or refractory leukemia: a pediatric oncology group study. Clin Cancer Res 11: 1884-1889

Jodrell DI, Bowman A, Rye R, Byrne B, Boddy A, Rafi I, Taylor GA, Johnston A, Clendeninn NJ (1999) A phase I study of the lipophilic thymidylate synthase inhibitor Thymitaq (nolatrexed dihydrochloride) given by 10-day oral administration. Br J Cancer 79: 915-920

Latz JE, Chaudhary AK, Ghosh A, Johnson RJ (2006) Population pharmacokinetic analysis of ten phase II clinical trials of pemetrexed in cancer patients. Cancer Chemother Pharmacol 57: 401-411

Lee PID, Amidon GL (1996) Pharmacokinetic Analysis. Lancaster: Technomic

Leveque D, Jehl F (1996) Clinical pharmacokinetics of vinorelbine. Clin Pharmacokinet 31: $184-197$

Li KM, Clarke SJ, Rivory LP (2003) Quantitation of plasma thymidine by high-performance liquid chromatography-atmospheric pressure chemical ionization mass spectrometry and its application to pharmacodynamic studies in cancer patients. Anal Chim Acta 486: 51-61

Li KM, Rivory LP, Clarke SJ (2005) Rapid quantitation of plasma $2^{\prime}$ deoxyuridine by high-performance liquid chromatography/atmospheric pressure chemical ionization mass spectrometry and its application to pharmacodynamic studies in cancer patients. J Chromatogr B 820: $121-130$

Li KM, Rivory LP, Hoskins J, Sharma R, Clarke SJ (2007) Altered deoxyuridine and thymidine in plasma following capecitabine treatment in colorectal cancer patients. Br J Clin Pharmacol 63: 67-74

Lucock MD, Daskalakis I, Schorah CJ, Lumb CH, Oliver M, Devitt H, Wild J, Dowell AC, Levene MI (1999) Folate-homocysteine interrelations: potential new markers of folate status. Mol Genet Metab 67: 23-35

Marquet P, Lachatre G, Debord J, Eichler B, Bonnaud F, Nicot G (1992) Pharmacokinetics of vinorelbine in man. Eur J Clin Pharmacol 42: $545-547$

Maybaum J, Cohen MB, Sadee W (1981) In vivo rates of pyrimidine nucleotide metabolism in intact mouse T-lymphoma (S-49) cells treated with 5-fluorouracil. J Biol Chem 256: 2126-2130

Mitchell F, Farrugia D, Rees C, Cunningham D, Judson I, Jackman AL (1997) Estimation of $2^{\prime}$-deoxyuridine (dUrd) in human plasma by HPLC with fluorimetric detection: a pharmacodynamic marker for thymidylate synthase (TS) inhibition. Br J Cancer 75: 25

Naurath HJ, Joosten E, Riezler R, Stabler SP, Allen RH, Lindenbaum J (1995) Effects of vitamin $B_{12}$, folate, and vitamin $B_{6}$ supplements in elderly people with normal serum vitamin concentrations. Lancet 346 $85-89$

Niyikiza C, Baker SD, Seitz DE, Walling JM, Nelson K, Rusthoven JJ, Stabler SP, Paoletti P, Calvert AH, Allen RH (2002) Homocysteine and methylmalonic acid: markers to predict and avoid toxicity from pemetrexed therapy. Mol Cancer Ther 1: 545-552

Rees C, Beale P, Trigo JM, Mitchell F, Jackman A, Smith R, Douglass E, Judson I (2003) Phase I trial of ZD9331, a nonpolyglutamatable thymidylate synthase inhibitor, given as a 5-day continuous infusion to patients with refractory solid malignancies. Clin Cancer Res 9: 2049-2055

Refsum H, Nygard O, Kvale G, Ueland PM, Vollset SE (1996) The Hordaland homocysteine study: the opposite tails odds ratios reveal differential effects of gender and intake of vitamin supplements at high and low plasma total homocysteine concentrations. J Nutr 126: 1244 S - 1248 S

Sabot C, Marquet P, Debord J, Carpentier N, Merle L, Lachatre G (1998) Bayesian pharmacokinetic estimation of vinorelbine in non-small-cell lung cancer patients. Eur J Clin Pharmacol 54: 171-175

Savage DG, Lindenbaum J, Stabler SP, Allen RH (1994) Sensitivity of serum methylmalonic acid and total homocysteine determinations for diagnosing cobalamin and folate deficiencies. Am J Med 96: 239-246

Shih C, Habeck LL, Mendelsohn LG, Chen VJ, Schultz RM (1998) Multiple folate enzyme inhibition; mechanisms of a novel pyrrolopyrimidine-based antifolate LY231514 (MTA). Adv Enzyme Regul 38: $135-152$

Smith PG, Marshman E, Calvert AH, Newell DR, Curtin NJ (1999) Prevention of thymidine and hypoxanthine rescue from MTA (LY231514) growth inhibition by dipyridamole in human lung cancer cell lines. Semin Oncol 26: 63-67

Valik D, Radina M, Sterba J, Vojtesek B (2004) Homocysteine: exploring its potential as a pharmacodynamic biomarker of antifolate chemotherapy. Pharmacogenomics 5: $1151-1162$

Worzalla JF, Shih C, Schultz RM (1998) Role of folic acid in modulating the toxicity and efficacy of the multitargeted antifolate, LY231514. Anticancer Res 18: 3235-3239 